\title{
Two-dimensional solutions for Born-Infeld fields
}

\section{Rafael Ferraro ${ }^{1}$}

Instituto de Astronomía y Fúsica del Espacio, Casilla de Correo 67, Sucursal 28, 1428 Buenos Aires, Argentina

Departamento de Física, Facultad de Ciencias Exactas y Naturales, Universidad de Buenos Aires, Ciudad Universitaria, Pabellón I, 1428 Buenos Aires, Argentina

E-mail: ferraro@iafe.uba.ar

ABSTRACT: The non-linear second order Born-Infeld equation is reduced to a simpler first order complex equation, which can be trivially solved for the coordinates as functions of the field. Each solution is determined by the choice of a holomorphic function subjected to boundary conditions. The explanation of the method is accompanied by applications to Born-Infeld electrostatics, magnetostatics and wave propagation.

Keywords: Integrable Equations in Physics, Integrable Field Theories

ARXiv EPRINT: 1304.5506

\footnotetext{
${ }^{1}$ Member of Carrera del Investigador Científico (CONICET, Argentina).
} 


\section{Contents}

1 Introduction 1

2 Born-Infeld Lagrangian 2

3 Two-dimensional Euclidean geometry 3

3.1 Complex potential 5

$\begin{array}{ll}3.2 & \text { Example: field between grounded conductors }\end{array}$

$\begin{array}{lll}3.3 & \text { Approximate solution } & 7\end{array}$

4 Born-Infeld electrodynamics $\quad 7$

4.1 Pure electric field 8

$\begin{array}{lr}4.2 & \text { Pure magnetic field } \\ 4.3 & \text { Stationary waves }\end{array}$

$\begin{array}{lll}4.3 & \text { Stationary waves } & 10\end{array}$

5 Conclusion $\quad 12$

\section{Introduction}

Born-Infeld equation,

$$
\left(1-b^{-2} c^{-2} u_{t}^{2}\right) u_{x x}+2 b^{-2} c^{-2} u_{t} u_{x} u_{t x}-\left(1+b^{-2} u_{x}^{2}\right) c^{-2} u_{t t}=0,
$$

( $u_{x}$ stands for $\partial_{x} u$, etc.) is a non-linear wave equation which is derived from the Lagrangian $L=\sqrt{1-b^{-2}\left(c^{-2} u_{t}^{2}-u_{x}^{2}\right)}$ and appears in several physical contexts. It descends from the Nambu-Goto action for a string in $2+1$ dimensions when a proper parametrization is chosen [1]. Besides, its solutions can be mapped into solutions of the Galileo-invariant Chaplygin gas in 1+1 dimensions, since Chaplygin gas is another descendent of the Nambu-Goto action [2,3]. Born-Infeld equation (1.1) also takes part in Born-Infeld electrodynamics [4, 5] when electromagnetic waves depending just on two variables are considered. Born-Infeld equation is integrable [6, 7] and has a multi-Hamiltonian structure [8]; the corresponding Cauchy problem is studied in ref. [9].

Eq. (1.1) is very close to the quasilinear elliptic equation

$$
\left(1+b^{-2} u_{y}^{2}\right) u_{x x}-2 b^{-2} u_{x} u_{y} u_{x y}+\left(1+b^{-2} u_{x}^{2}\right) u_{y y}=0 .
$$

This equation was firstly obtained by Lagrange in 1762 when he studied the problem of minimizing the area of a surface whose boundary is a given closed curve in $\mathbb{R}^{3}$ (Plateau's problem) $[10,11]$; such problem is a natural generalization of the problem of geodesics. In fact, the minimal surface equation (1.2) comes from the Lagrangian $L=\sqrt{1+b^{-2}\left(u_{x}^{2}+u_{y}^{2}\right)}$, 
so the action is the area of the surface $\zeta=b^{-1} u(x, y)$ ( $\zeta$ is the third Cartesian coordinate). ${ }^{1}$ Eq. (1.2) says that minimal surfaces have vanishing mean curvature. The solutions of eq. (1.2) can be expressed through a parametric representation where each solution is determined by the choice of a pair of related complex functions (Weierstrass-Enneper parametrization [12]). The Dirichlet problem for the eq. (1.2) is studied in ref. [13].

Eq. (1.2) could be regarded as a deformed Laplace equation. Also the equation

$$
\left(1-b^{-2} u_{y}^{2}\right) u_{x x}+2 b^{-2} u_{x} u_{y} u_{x y}+\left(1-b^{-2} u_{x}^{2}\right) u_{y y}=0,
$$

which appears in two-dimensional Born-Infeld electrostatics, is a deformed Laplace equation. Eq. (1.3) is derived from the Lagrangian $L=\sqrt{1-b^{-2}\left(u_{x}^{2}+u_{y}^{2}\right)}$. Their solutions were characterized by Pryce $[14,15]$ through a complex method where each solution is associated with a holomorphic function (see also References [16-18]). Eq. (1.3) is the equation for maximal surfaces, which are space-like surfaces in $(2+1)$ Minkowski space with vanishing mean curvature. Maximal surfaces defined on a domain $D$ of the complex plane $\varsigma$ also admit a Weierstrass-Enneper parametrization [19]:

$$
(x(\varsigma), y(\varsigma), \zeta(\varsigma))=\operatorname{Re} \int\left(\frac{1}{2} f\left(1+g^{2}\right), \frac{i}{2} f\left(1-g^{2}\right),-f g\right) d \varsigma,
$$

where $f$ is holomorphic and $g$ is meromorphic on $D$ such that $f g^{2}$ is holomorphic on $D$ and $|g(\varsigma)| \neq 1$ for $\varsigma \in D$.

Since the solutions of each one of the equations (1.1), (1.2) and (1.3) can be transformed into the others by properly renaming the variables and $b^{2}$, we will focus just on the eq. (1.3). In the following sections we will explain the method for finding the solutions of eq. (1.3). We will show in a few steps that the equation governing the two-dimensional electrostatic Born-Infeld field can be put into the compact form (3.6), where $e$ is an auxiliary complex field associated with the real 1-form $\mathbf{E} \equiv d u$, and $z, \bar{z}$ are complex coordinates $(z=x+i y)$. In section 4 we will connect the solutions of eqs. (1.1), (1.2) and (1.3) to field configurations of Born-Infeld electrodynamics.

\section{Born-Infeld Lagrangian}

The Born-Infeld Lagrangian density for a scalar field is

$$
\mathcal{L}[u]=\sqrt{|g|} \sqrt{1-b^{-2} g^{k j} \partial_{k} u \partial_{j} u}
$$

So the Lagrange equation is

$$
\partial_{i}\left(\frac{\sqrt{|g|} g^{i j} \partial_{j} u}{\sqrt{1-b^{-2} g^{k j} \partial_{k} u \partial_{j} u}}\right)=0 .
$$

\footnotetext{
${ }^{1}$ Vectors $\overrightarrow{\delta A}=\left(d x, 0, b^{-1} u_{x} d x\right)$ and $\delta \vec{B}=\left(0, d y, b^{-1} u_{y} d y\right)$ are tangent to the surface $\zeta=u(x, y)$. Thus, the vector product $\overrightarrow{\delta A} \times \overrightarrow{\delta B}$ defines the area of the surface immersed in $\mathbb{R}^{3}$. The infinitesimal area then is $|\overrightarrow{\delta A} \times \delta \vec{B}|=\sqrt{1+b^{-2}\left(u_{x}^{2}+u_{y}^{2}\right)} d x d y$, which leads to the action for the eq. (1.2).
} 
We introduce the related 1 -forms

$$
E_{j} \equiv \partial_{j} u, \quad D_{j} \equiv \frac{E_{j}}{\sqrt{1-b^{-2} g^{k l} E_{k} E_{l}}} .
$$

According to eq. (2.2) the field $D_{j}$ accomplishes the equation

$$
\partial_{i}\left(\sqrt{|g|} g^{i j} D_{j}\right)=0 .
$$

In geometric notation, the dynamics of the field is summarized in the equations ${ }^{2}$

$$
d \mathbf{E}=0, \quad d * \mathbf{D}=0,
$$

where the 1-forms $\mathbf{E}$ and $\mathbf{D}$ accomplish the constitutive relation

$$
\mathbf{D} \equiv \frac{\mathbf{E}}{\sqrt{1-b^{-2}\|\mathbf{E}\|^{2}}} .
$$

One should solve the system (2.5), (2.6) and then retrieve the scalar potential $u$ from $\mathbf{E}=d u$.

Remarkably, the constitutive relation (2.6) is automatically fulfilled if $\mathbf{E}$ and $\mathbf{D}$ are written in the suggestive form

$$
\mathbf{E}=\frac{\mathbf{e}}{1+\frac{\|\mathbf{e}\|^{2}}{4 b^{2}}}, \quad \mathbf{D}=\frac{\mathbf{e}}{1-\frac{\|\mathbf{e}\|^{2}}{4 b^{2}}},
$$

where $\mathbf{e}$ is an auxiliary 1-form field. By replacing eq. (2.7) in eq. (2.5) one gets two equations for e:

$$
\begin{array}{r}
\left(1+\frac{\|\mathbf{e}\|^{2}}{4 b^{2}}\right) d \mathbf{e}-d\left(\frac{\|\mathbf{e}\|^{2}}{4 b^{2}}\right) \wedge \mathbf{e}=0, \\
\left(1-\frac{\|\mathbf{e}\|^{2}}{4 b^{2}}\right) d * \mathbf{e}+d\left(\frac{\|\mathbf{e}\|^{2}}{4 b^{2}}\right) \wedge * \mathbf{e}=0 .
\end{array}
$$

\section{Two-dimensional Euclidean geometry}

In two-dimensional Euclidean geometry,

$$
d s^{2}=d x^{2}+d y^{2}, \quad g_{i j}=\operatorname{diag}(1,1),
$$

the second order equation (2.2) becomes the eq. (1.3). With regard to the equivalent system of first order equations (2.8), (2.9), we can take advantage of the fact that both $\mathbf{e}$ and $* \mathbf{e}$ are 1 -forms if $n=2$. Thus, we can condensate these equations in a sole complex equation

$$
d(\mathbf{e}+i * \mathbf{e})+\frac{\|\mathbf{e}\|^{2}}{4 b^{2}} d(\mathbf{e}-i * \mathbf{e})-d\left(\frac{\|\mathbf{e}\|^{2}}{4 b^{2}}\right) \wedge(\mathbf{e}-i * \mathbf{e})=0,
$$

\footnotetext{
${ }^{2} *$ is the Hodge operator which converts the 1 -form $\mathbf{D}$ into a $(n-1)$-form $\left(n\right.$ is the dimension). If $\alpha_{i_{1} \ldots \ldots . i_{p}}$ are the components of the $p$-form $\alpha$ then $* \alpha_{\mu_{p+1} \ldots \ldots . \mu_{n}}=\frac{1}{p !} \sqrt{\left|\operatorname{det}\left(g_{\mu \nu}\right)\right|} \varepsilon_{\mu_{1} \ldots \ldots \mu_{p} \mu_{p+1} \ldots \ldots \mu_{n}} \alpha^{\mu_{1} \ldots \ldots . . \mu_{p}}$ where $\varepsilon$ is the Levi-Civita symbol whose value is $1(-1)$ for even (odd) permutations of the natural order of its indexes and vanishes for repeated indexes.
} 
Notice that the complex 1 -form $\mathbf{e}-i * \mathbf{e}$ is quite elemental in the coordinate basis $(d z, d \bar{z})$, where $z=x+i y$ :

$$
\mathbf{e}-i * \mathbf{e}=\left(e_{x} d x+e_{y} d y\right)-i\left(e_{y} d x-e_{x} d y\right)=\left(e_{x}-i e_{y}\right) d z .
$$

We will call

$$
e \equiv e_{x}-i e_{y}
$$

Therefore, the field equation (3.2) becomes

$$
-e d\left(\frac{|e|^{2}}{4 b^{2}}\right) \wedge d z+d(\bar{e} d \bar{z})+\frac{|e|^{2}}{4 b^{2}} d(e d z)=0
$$

where we replaced $\|\mathbf{e}\|^{2}=e_{x}^{2}+e_{y}^{2}=|e|^{2}=e \bar{e}$. Eq. (3.5) simplifies to

$$
d \bar{e} \wedge d \bar{z}=\frac{e^{2}}{4 b^{2}} d \bar{e} \wedge d z
$$

This complex equation summarizes the dynamics of the field. It can be tackled from a double perspective. If the auxiliary field $e$ is regarded as a function of $(z, \bar{z})$ (i.e., as a function of $(x, y))$, then one gets

$$
\frac{\partial \bar{e}}{\partial z}=-\frac{e^{2}}{4 b^{2}} \frac{\partial \bar{e}}{\partial \bar{z}}
$$

Instead, if the coordinate $z$ is regarded as a function of $(e, \bar{e})$ one obtains

$$
\frac{\partial \bar{z}}{\partial e}=\frac{e^{2}}{4 b^{2}} \frac{\partial z}{\partial e} .
$$

Remarkably, this last form is linear in $z(e, \bar{e})$, and can be rewritten as

$$
\frac{\partial \bar{z}}{\partial \xi}=-\frac{\partial z}{\partial(1 / \xi)}, \quad \xi \equiv \frac{e}{2 b}
$$

The general solution has the form

$$
z=f(1 / \xi)+g(\bar{\xi})
$$

with

$$
g^{\prime}(\xi)=-f^{\prime}(1 / \xi)
$$

(the prime means derivative with respect to the argument).

Eq. (2.5) says that $\mathbf{D}$ is singular when $\|\mathbf{E}\|=b$ (i.e., when $|\xi|=1$ ). Let us study the behavior of the solution (3.10), (3.11) at these values of the field. We want to know whether $|\xi|=1$ happens at isolated points or not. For this, we will evaluate $d z$ at $\xi=\exp [i \theta]$; since

$$
d z=f^{\prime}(1 / \xi) d(1 / \xi)+g^{\prime}(\bar{\xi}) d(\bar{\xi})=-g^{\prime}(\xi) d(1 / \xi)+g^{\prime}(\bar{\xi}) d(\bar{\xi}),
$$

then,

$$
\left.d z\right|_{\xi=\exp [i \theta]}=\left[-g^{\prime}(\exp [i \theta])+g^{\prime}(\exp [-i \theta])\right] d(\exp [-i \theta]) .
$$


Therefore the singularities occurs at isolate points (i.e., $d z=0$ ) whenever it is

$$
\operatorname{Im}\left[g^{\prime}(\xi)\right]_{|\xi|=1}=0 .
$$

In ref. [18], the reality condition (3.14) has been carried out by choosing functions $g^{\prime}$ that do not change under the transformation $\xi \longrightarrow 1 / \xi$ (for instance, any function with real coefficients that depends just on $\left.\xi^{\alpha}+1 / \xi^{\alpha}\right)$. In fact, $\xi$ and $1 / \xi$ are complex conjugate if $\xi=\exp [i \theta]$; so, such functions are automatically real on the circle $|\xi|=1$. Even so, it could happens that $\operatorname{Im}\left[g^{\prime}(\exp [i \theta])\right]$ results ill-defined for some values of $\theta$. In such cases $d z$ in eq. (3.13) could be non-null for such particular field directions (see the multipolar structures in ref. [18]). If $g^{\prime}(1 / \xi)=g^{\prime}(\xi)$, then the eq. (3.11) means that $f^{\prime}(\xi)=-g^{\prime}(\xi)$; so the solution of eq. (3.9) becomes

$$
z=-g(1 / \xi)+g(\bar{\xi})+\text { constant }
$$

where $g(\xi)$ is any holomorphic function such as

$$
g^{\prime}(\xi)=g^{\prime}(1 / \xi)
$$

The choice of $g(\xi)$ is constrained by boundary conditions; for instance, it can be required that $e$ goes to zero for $z$ going to infinity. Moreover, in ref. [18] it is shown that the singlevaluedness of the solution $z(\xi, \bar{\xi})$ and the isolation of the singular points are guaranteed by functions $g^{\prime}(\xi)$ which do not possess branch cuts reaching the circle $|\xi|=1$.

\subsection{Complex potential}

The scalar field $u$ is a potential for the field $\mathbf{E}$. Besides, the closed 1-form $* \mathbf{D}$ (see eq. (2.5)) can be also associated with a potential $v$,

$$
* \mathbf{D} \equiv-d v .
$$

Then, the complex potential

$$
w \equiv u+i v
$$

satisfies

$$
d w=\mathbf{E}-i * \mathbf{D} .
$$

Using eqs. (2.7) and (3.3) one obtains

$$
(2 b)^{-1} d w=\frac{\xi d z-|\xi|^{2} \bar{\xi} d \bar{z}}{1-|\xi|^{4}} .
$$

Eq. (3.6) implies that $d e \wedge d w=0$, which means that $w$ is a holomorphic function of $e$ : $w=w(e)$. In fact, using eq. (3.15) one gets

$$
d w=\frac{2 b}{\xi} g^{\prime}(\xi) d \xi
$$

Since the function $g^{\prime}(\xi)$ accomplishes the eq. (3.16), the complex potential satisfies

$$
w(\xi)=-w(1 / \xi)+\text { constant } .
$$




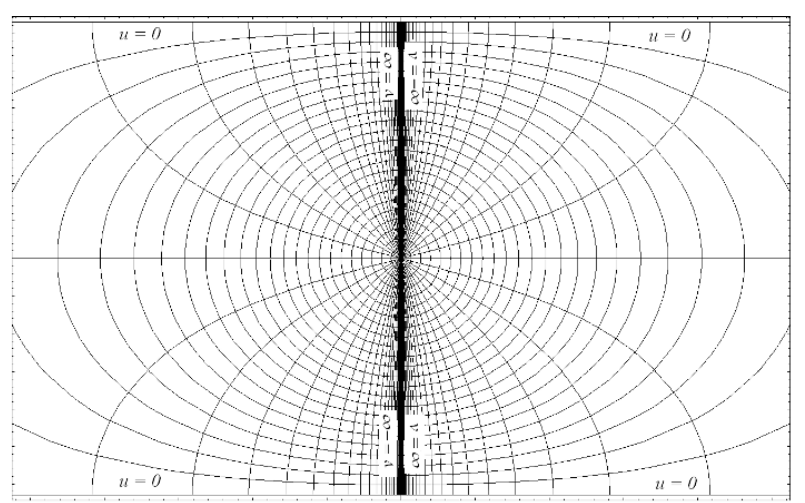

Figure 1. Equipotential lines and field lines for the field of eq. (3.26).

\subsection{Example: field between grounded conductors}

As explained in the previous subsection, each solution $u(x, y)=R e[w]$ of eq. (1.3) is determined by the choice of the holomorphic function $g^{\prime}(\xi)$ or, alternatively, the holomorphic function $w(\xi)$; both functions are related by the eq. (3.21). We will illustrate the procedure with an example differing from those considered in ref. [18]. Let us choose the function

$$
g^{\prime}(\xi)=\frac{d}{\pi} \frac{\xi}{1+\xi^{2}}
$$

where $d$ is a constant with units of distance. $g^{\prime}(\xi)$ accomplishes the eq. (3.16). The complex potential in eq. (3.21) becomes

$$
w=\frac{2 b d}{\pi} \arctan [\xi]
$$

Thus the solution (3.15) yields

$$
\frac{2 \pi}{d} z=\log \left[\frac{1+\bar{\xi}^{2}}{1+1 / \xi^{2}}\right] .
$$

This result can be solved for $\xi^{2}$ :

$$
\xi^{2}=-\frac{\exp \left[\frac{2 \pi}{d} \bar{z}\right]-1}{\exp \left[-\frac{2 \pi}{d} z\right]-1} .
$$

This field reaches the maximum value $|\xi|=1$ on the $y$-axis. In fact if $z=i y$ one gets $\xi^{2}=-1$. The fact that the maximum value is reached not at an isolated point but on a line is due to the poles the function $g^{\prime}(\xi)$ displays on the circle $|\xi|=1$.

We can also realize that the lines $y= \pm d / 2$ are equipotential lines. In fact, by replacing $z=x \pm i d / 2$ one gets $\xi^{2}<0$ :

$$
\xi^{2}=-\frac{\exp \left[\frac{2 \pi}{d} x\right]+1}{\exp \left[-\frac{2 \pi}{d} x\right]+1} ;
$$


this means that the field is $\xi=-i \xi_{y}$, so it is normal to the lines $y= \pm d / 2$. One can check in eq. (3.24) that $u=R e[w]$ vanishes when $\xi$ is pure imaginary. To make a figure of the equipotential lines, we replace $\xi(w)=\tan [w \pi /(2 b d)]$ in eq. (3.25), with $w=u_{o}+i v$; then $v$ plays the role of a parameter for the line $u=u_{o}$. Some equipotential and field lines are shown in figure 1. Actually, only the $x \leq 0$ region should be considered in the solution (3.26) since $|\xi|>1$ for $x>0$. However, in figure 1 the field has been continuously extended to the semi-space $x>0$, by choosing $g^{\prime}(\xi)=-(d / \pi) \xi /\left(1+\xi^{2}\right)$ in this region. The change of sign in $g^{\prime}(\xi)$ implies the change $z \longrightarrow-z$ in the solution (3.26) and a change of sign in the expression (3.24) for the complex potential.

Figure 1 is the field of a point-like charge between two parallel grounded conductors separated by a distance $d$. It could also be regarded as the field of a succession of alternating charges at a distance $d$ on the $y$-axis. These image charges are joined by lines of maximum field, as it happens in the multipolar solutions studied in ref. [18]. The fact that $v$ ranges between $-\infty$ and $\infty$ implies that the point-like charge at the origin is infinite, which is also a characteristic of the multipolar solutions. ${ }^{3}$

The field $\mathbf{E}(x, y)=d u$ can be computed by differentiating $u=R e[w(\xi(z, \bar{z}))]$ or directly replacing $\mathbf{e}$ in eq. (2.7) with the result (3.26) for the complex auxiliary field.

\subsection{Approximate solution}

In general, we will hardly invert eq. (3.15) to get an expression like (3.26) for the field $\xi(z, \bar{z})$. However we could approach the field $\xi(z, \bar{z})$ by iterating the solution of eq. (3.7). At the lowest order in $b^{-2}$ the solution is $e=F(z)$, where $F$ is an analytic function. It is easy to check that the following order is

$$
e(z, \bar{z})=F(z)-\frac{1}{4 b^{2}} \frac{\partial F(z)}{\partial z} \int F(\bar{z})^{2} d \bar{z}+\mathcal{O}\left(b^{-4}\right) .
$$

At this order of approximation, the complex potential $w(z, \bar{z})$ is obtained from eq. (3.20):

$$
d w=e d z-\frac{\bar{e}|e|^{2}}{4 b^{2}} d \bar{z}+\mathcal{O}\left(b^{-4}\right) .
$$

Therefore

$$
w(z, \bar{z})=\int F(z) d z-\frac{1}{4 b^{2}} F(z) \int F(\bar{z})^{2} d \bar{z}+\mathcal{O}\left(b^{-4}\right)
$$

\section{Born-Infeld electrodynamics}

Born-Infeld electrodynamics is a non-linear extension of Maxwell electromagnetism $[4,5]$. In both theories the electromagnetic field is an exact 2-form $F=d A$ in Minkowski spacetime, where the 1-form $A$ is the electromagnetic potential; so it is $d F=0$. But, differing from Maxwell's field, Born-Infeld electromagnetic field is governed by the dynamical equations

$$
d\left(\frac{* F+\frac{P}{b^{2}} F}{\sqrt{1+\frac{2 S}{b^{2}}-\frac{P^{2}}{b^{4}}}}\right)=0,
$$

\footnotetext{
${ }^{3}$ The charge is the flux of $* \mathbf{D}: 2 \pi Q=\oint D_{x} d y-D_{y} d x=\oint d v$.
} 
where $S$ and $P$ are the invariants

$$
\begin{aligned}
S & \equiv \frac{1}{4} F_{i j} F^{i j}=\frac{1}{2}\left(|\vec{B}|^{2}-|\vec{E}|^{2}\right), \\
P & \equiv \frac{1}{4} * F_{i j} F^{i j}=\vec{E} \cdot \vec{B} .
\end{aligned}
$$

The dynamical equations can be derived from the Lagrangian density

$$
\mathcal{L}[A]=\frac{b^{2}}{4 \pi} \sqrt{|g|}\left(1-\sqrt{1+\frac{2 S}{b^{2}}-\frac{P^{2}}{b^{4}}}\right) .
$$

Notice that Maxwell's theory is recovered in the limit $b \longrightarrow \infty$. Born-Infeld Lagrangian (4.4) is exceptional because, together with another unphysical Lagrangian, is the only function of $S$ and $P$ ensuring the absence of birefringence and shock waves [20-23].

Except for the field of a point-like charge [5] and the essentially two-dimensional solutions we are going to show in this section, it is very hard to find exact solutions for Born-Infeld electrodynamics. Maxwell's plane waves are trivial solutions because they have vanishing invariants $S$ and $P$; so no difference remains between Maxwell and Born-Infeld equations in such case. The exact solution for a plane wave interacting with a static uniform field has been obtained in ref. [24]. The case for a cylindrical wave has been recently worked out [25]. Stationary solutions were studied under the form of perturbative series [26]; the uniqueness of such solutions was also examined [27]. It has been shown that Born-Infeld dynamics can be thrown into a form similar to MHD equations by promoting the Poynting vector and the energy to the status of unknown variables [28]. The chance of detecting effects of Born-Infeld electrodynamics in laser-plasma experiments is analyzed in refs. [29, 30].

As we will show in this section, the eqs. (1.1), (1.2) and (1.3) describe some Born-Infeld field configurations with $P=0$. By replacing $P=0$ in eq. (4.1), the dynamical equations become

$$
\left(1+b^{-2} 2 S\right) d * F-b^{-2} d S \wedge * F=0
$$

\subsection{Pure electric field}

Let us consider the electromagnetic potential

$$
A=u(x, y) c d t
$$

Then $F=u_{x} c d t \wedge d x+u_{y} c d t \wedge d y$ is an electrostatic field whose field lines lie on the $(x, y)$-plane. The electric components of the field are $F_{0 \alpha}=E_{\alpha}=\partial_{\alpha} u$. Thus,

$$
\begin{aligned}
& 2 S=-u_{x}^{2}-u_{y}^{2}, \\
& * F=u_{x} d y \wedge d \zeta-u_{y} d x \wedge d \zeta
\end{aligned}
$$

( $\zeta$ is the third Cartesian coordinate). In this case the dynamical equation (4.5) turns out to be the eq. (1.3). This is because the Lagrangian (4.4) is essentially $\sqrt{1-b^{-2}|\vec{E}|^{2}}$, so it coincides with the Lagrangian in section 3. 
The simplest example is the cylindrically symmetric field associated with a charge density $\lambda$ distributed along the $\zeta$-axis. According to the definition of $e$ (see eq. (3.4)), such a radial symmetry requires that $\arg (z)=-\arg (\xi)$. Then, $g(\xi)$ in eq. (3.15) has to be linear; thus $g^{\prime}(\xi)$ is a real constant and accomplishes the reality condition (3.14). The value of the constant $g^{\prime}$ is dictated by the Coulombian limit $b \rightarrow \infty$; we will see that $g^{\prime}=\lambda /(2 b)$. Therefore the cylindrically symmetric solution (3.15) is

$$
z=\frac{\lambda}{2 b}\left(\frac{1}{\xi}-\bar{\xi}\right)
$$

In this case, the function $z(\xi, \bar{\xi})$ is easily inverted to obtain

$$
\xi(z, \bar{z})=\frac{b}{\lambda} \bar{z}\left(\sqrt{1+\frac{\lambda^{2}}{b^{2} z \bar{z}}}-1\right)=\frac{\lambda}{b z}\left(\sqrt{1+\frac{\lambda^{2}}{b^{2} z \bar{z}}}+1\right)^{-1} .
$$

To compute the potential $u(x, y)$, let us integrate the eq. (3.21) for $g^{\prime}=\lambda /(2 b)$; it results

$$
w(\xi)=-\lambda \log [\xi] .
$$

Then, by replacing the expression (4.10) one gets

$$
\begin{aligned}
u(x, y) & =\operatorname{Re}\left\{\lambda \log \left[\frac{\lambda}{b(x+i y)}\right]-\lambda \log \left[\sqrt{1+\frac{\lambda^{2}}{b^{2}\left(x^{2}+y^{2}\right)}}+1\right]\right\} \\
& =-\lambda \log \left[\sqrt{b^{2} \lambda^{-2} r^{2}+1}+|\lambda|^{-1} b r\right]
\end{aligned}
$$

where $r^{2}=x^{2}+y^{2}$. When $b \longrightarrow \infty$ one recovers the Coulombian potential $u=-\lambda \log [r]+$ constant.

\subsection{Pure magnetic field}

The electromagnetic potential

$$
A=u(x, y) d \zeta
$$

( $\zeta$ is the third Cartesian coordinate) leads to a pure magnetic field whose field lines lie on the $(x, y)$-plane. In fact, the field $F$ is deprived of components $F_{0 \alpha}: F=u_{x} d x \wedge d \zeta+u_{y} d y \wedge d \zeta$. Since $B_{\alpha}=\epsilon_{\alpha \beta \gamma} F^{\beta \gamma}$, then it is $B_{x}=u_{y}$ and $B_{y}=-u_{x}$. Thus,

$$
\begin{aligned}
& 2 S=u_{x}^{2}+u_{y}^{2}, \\
& * F=-u_{x} c d t \wedge d y+u_{y} c d t \wedge d x .
\end{aligned}
$$

The dynamical equation (4.5) becomes eq. (1.2), which differs from eq. (1.3) in the sign of $b^{-2}$; this is because now the Lagrangian is basically $\sqrt{1+b^{-2}|\vec{B}|^{2}}$ instead of $\sqrt{1-b^{-2}|\vec{E}|^{2}}$. Any solution $u(x, y)$ obtained through the procedure explained in the previous section can be converted in a solution of eq. (1.2) by changing $b^{2} \longrightarrow-b^{2}$. Alternatively, one can also change $x \longrightarrow i x, y \longrightarrow i y$, which is equivalent to change $z \longrightarrow i z$ and $\bar{z} \longrightarrow i \bar{z}$ in $u(z, \bar{z})=\operatorname{Re}[w(z, \bar{z})]$. 
For instance, according to eq. (4.12) the potential for a neutral straight steady current has the form

$$
u=-\lambda \log \left[\sqrt{b^{2} \lambda^{-2} r^{2}-1}+\sqrt{b^{2} \lambda^{-2} r^{2}}\right]=-\lambda\left|\operatorname{arccosh}\left[\frac{b r}{\lambda}\right]\right|,
$$

which represents a catenoid in $\mathbb{R}^{3}$ (a well known minimal surface [11]). The field $F=$ $(d u / d r) d r \wedge d \zeta$ diverges at $r=b^{-1} \lambda$. This result could mean that pure Born-Infeld magnetostatic fields are just an approximation to be used far from the sources. Near to the sources one should not ignore the true nature of the charges that constitute the steady current.

Notice that the replacement $b^{2} \longrightarrow-b^{2}$ in the Lagrangian (2.1) amounts the exchange of roles between $\mathbf{E}$ and $\mathbf{D}$ in eq. (2.7). Thus, the association between the Born-Infeld magnetostatic field $B_{x}=u_{y}, B_{y}=-u_{x}$ and the complex field $e$ turns out to be

$$
-B_{y}-i B_{x}=u_{x}-i u_{y}=\frac{2 b}{\frac{2 b}{e}-\frac{\bar{e}}{2 b}} .
$$

The auxiliary field $e(z, \bar{z})$ is now governed by the equation

$$
\frac{\partial \bar{z}}{\partial e}+\frac{e^{2}}{4 b^{2}} \frac{\partial z}{\partial e}=0
$$

whose general solution is

$$
z=f(1 / \xi)-g(\bar{\xi}), \quad \text { where } \quad-g^{\prime}(\xi)=f^{\prime}(1 / \xi)
$$

According to eq. (4.17), $\vec{B}$ is singular where $|\xi|=1$.

\subsection{Stationary waves}

Let us start with the electromagnetic potential

$$
A=u(x, t) d y \text {. }
$$

The field has now electric and magnetic orthogonal components, $F=c^{-1} u_{t} c d t \wedge d y+$ $u_{x} d x \wedge d y$. Then, $E_{y}=c^{-1} u_{t}$ and $B_{\zeta}=u_{x}$. Therefore,

$$
\begin{aligned}
& 2 S=u_{x}^{2}-c^{-2} u_{t}^{2}, \\
& * F=c^{-1} u_{t} d x \wedge d \zeta+u_{x} c d t \wedge d \zeta .
\end{aligned}
$$

In this case the dynamical equation (4.5) yields the Born-Infeld equation (1.1). This equation becomes eq. (1.3) by replacing $y \longrightarrow i c t$ and $b \longrightarrow i b$.

We will look for stationary waves between two parallel conductors. We will apply the expression (3.28) to get approximate solutions. Stationary waves can be obtained by starting from the holomorphic function $F(z)=e_{o} \cos k z$. In fact, according to eq. (3.30) this choice implies the potential

$$
u=\operatorname{Re}[w]=\left.\operatorname{Re}\left[-\frac{e_{o}}{k} \sin k z\right]\right|_{y=i c t}+\mathcal{O}\left(b^{-2}\right)=-\frac{e_{o}}{k} \cos k c t \sin k x+\mathcal{O}\left(b^{-2}\right) .
$$


So, for $b \longrightarrow \infty$ one obtains the Maxwellian potential for stationary waves. Let us use the eq. (3.28) to compute the next order of approximation:

$$
e(z, \bar{z})=e_{o} \cos k z+\frac{e_{o}^{3}}{16 b^{2}} \sin k z(2 k \bar{z}+\sin 2 k \bar{z})+\mathcal{O}\left(b^{-4}\right) .
$$

Although we expected an oscillating solution, the approach (3.28) produced a secular term $2 k \bar{z}$. This means that the result (4.24) is valid just for $k|z| e_{o}^{2} b^{-2} \ll 1$. The secular term can be healed by replacing $k \bar{z} e_{o}^{2} b^{-2} / 8$ with $\sin \left(k \bar{z} e_{o}^{2} b^{-2} / 8\right)$. In fact, it is easy to verify that the field

$$
\begin{aligned}
e(z, \bar{z}) & =e_{o} \cos k z+e_{o} \sin k z \sin \frac{e_{o}^{2} k \bar{z}}{8 b^{2}}+\frac{e_{o}^{3}}{16 b^{2}} \sin k z \sin 2 k \bar{z}+\mathcal{O}\left(b^{-4}\right) \\
& =e_{o} \cos k\left(z-\frac{e_{o}^{2}}{8 b^{2}} \bar{z}\right)+\frac{e_{o}^{3}}{16 b^{2}} \sin k z \sin 2 k \bar{z}+\mathcal{O}\left(b^{-4}\right)
\end{aligned}
$$

accomplishes the eq. (3.7) at the considered order of approximation. The secular term then expresses a correction to the frequency of the stationary wave.

We can use the eq. (3.30) to compute the complex potential $w(z, \bar{z})$. After healing the secular term, we get the complex potential fulfilling the eq. (3.29) for the field (4.25):

$$
\begin{aligned}
w(z, \bar{z}) & =\frac{e_{o}}{k} \sin k z-\frac{e_{o}}{k} \cos k z \sin \frac{e_{o}^{2} k \bar{z}}{8 b^{2}}-\frac{e_{o}^{3}}{16 k b^{2}} \cos k z \sin 2 k \bar{z}+\mathcal{O}\left(b^{-4}\right) \\
& =\frac{e_{o}}{k} \sin k\left(z-\frac{e_{o}^{2}}{8 b^{2}} \bar{z}\right)-\frac{e_{o}^{3}}{16 k b^{2}} \cos k z \sin 2 k \bar{z}+\mathcal{O}\left(b^{-4}\right)
\end{aligned}
$$

We now get the real potential $u(x, y)=R e[w]$ that accomplishes the eq. (1.3), and pass to the solution of Born-Infeld equation (1.1) by changing $y \longrightarrow i c t$ and $b \longrightarrow i b$ :

$$
\begin{aligned}
u(x, t)= & \frac{e_{o}}{2 k} \sin k\left(x-c t+\frac{e_{o}^{2}}{8 b^{2}}(x+c t)\right)+\frac{e_{o}}{2 k} \sin k\left(x+c t+\frac{e_{o}^{2}}{8 b^{2}}(x-c t)\right)(4.27) \\
& +\frac{e_{o}^{3}}{32 k b^{2}} \cos k(x-c t) \sin 2 k(x+c t)+\frac{e_{o}^{3}}{32 k b^{2}} \cos k(x+c t) \sin 2 k(x-c t)+\mathcal{O}\left(b^{-4}\right) .
\end{aligned}
$$

At the considered order of approximation, the result can be reorganized as

$$
\begin{aligned}
u(x, t) & =\frac{e_{o}}{k} \sin \left[\left(1+\frac{e_{o}^{2}}{8 b^{2}}\right) k x\right] \cos \left[\left(1-\frac{e_{o}^{2}}{8 b^{2}}\right) k c t\right]+\frac{e_{o}^{3}}{16 k b^{2}} \sin k x \cos k c t(\cos 2 k x+\cos 2 k c t)+\mathcal{O}\left(b^{-4}\right) \\
& =\frac{e_{o}}{k} \sin \left[\left(1+\frac{e_{o}^{2}}{8 b^{2}}\right) k x\right] \cos \left[\left(1-\frac{e_{o}^{2}}{8 b^{2}}\right) k c t\right]\left(1+\frac{e_{o}^{2}}{16 b^{2}}(\cos 2 k x+\cos 2 k c t)\right)+\mathcal{O}\left(b^{-4}\right) .
\end{aligned}
$$

To fulfill boundary conditions $u(0, t)=0$ and $u(d, t)=0$, corresponding to two parallel grounded conductors at a distance $d$, we choose

$$
k=\frac{n \pi}{d}\left(1-\frac{e_{o}^{2}}{8 b^{2}}\right)+\mathcal{O}\left(b^{-4}\right) .
$$

Then, as a consequence of the non-linearity, the resonant frequencies in a cavity depend on the amplitude.

The obtained solution can be boosted along the parallel conductors to get propagating waves in a waveguide [31, 32]. 


\section{Conclusion}

We have shown a method to build solutions of Born-Infeld equation (1.1) and its relatives (1.2), (1.3). The method exploits the power of exterior calculus in the complex basis of $\mathbb{R}^{2}$, which is the natural language for this problem. Although the method was developed for the eq. (1.3), the obtained solutions are converted into solutions to the other equations by properly changing the variables or the Born-Infeld constant $b^{2}$.

Remarkably, eq. (1.3) becomes Laplace equation at the points where the first derivatives $u_{x}, u_{y}$ vanish. This distinctive feature prevents the existence of smooth extremes in static Born-Infeld configurations. In fact, Laplace equation could not be fulfilled at an extreme since $u_{x x}$ and $u_{y y}$ should have the same sign. This means that Born-Infeld dynamics does not harbor smooth static solutions going to zero at the boundaries (i.e., no solitary waves exist other than those traveling at the speed of light). These aspects of extremes in Born-Infeld electrostatics can be recognized in the examples shown in sections 3.2 and 4.1.

Since eq. (3.6) is linear in $z$, the general solution (3.15) expresses $z$ as a function of the auxiliary complex field. In most of the cases, it will be very hard to invert this function for obtaining the field as a function of the coordinates. However, since non-linear effects are expected to be very weak, just an expression at the lowest order in $b^{-2}$ would be enough for experimental tests. This is the case of the approximate expressions (3.28) and (3.30) that we applied in section 4.3 to study Born-Infeld stationary waves in a cavity.

\section{References}

[1] M. Bordemann and J. Hoppe, The dynamics of relativistic membranes. 2. Nonlinear waves and covariantly reduced membrane equations, Phys. Lett. B 325 (1994) 359 [hep-th/9309025] [INSPIRE].

[2] M. Bordemann and J. Hoppe, The dynamics of relativistic membranes. 1. Reduction to two-dimensional fluid dynamics, Phys. Lett. B 317 (1993) 315 [hep-th/9307036] [INSPIRE].

[3] R. Jackiw and A. Polychronakos, Fluid dynamical profiles and constants of motion from D-branes, Commun. Math. Phys. 207 (1999) 107 [hep-th/9902024] [INSPIRE].

[4] M. Born and L. Infeld, Foundations of the new field theory, Nature 132 (1933) 1004.

[5] M. Born and L. Infeld, Foundations of the new field theory, Proc. Roy. Soc. Lond. A 144 (1934) 425 [inSPIRE].

[6] B.M. Barbashov and N.A. Chernikov, Solution of the two plane wave scattering problem in a nonlinear scalar field theory of the Born-Infeld type, Sov. Phys. JETP 24 (1967) 437.

[7] D. Fairlie and J. Mulvey, Integrable generalizations of the two-dimensional Born-Infeld equation, J. Phys. A 27 (1994) 1317 [hep-th/9310005] [INSPIRE].

[8] M. Arik, F. Neyzi, Y. Nutku, P. Olver and J. Verosky, Multi-Hamiltonian structure of the Born-Infeld equation, J. Math. Phys. 30 (1989) 1338.

[9] B.M. Barbashov and N.A. Chernikov, Solution and quantization of a nonlinear two-dimensional model for a Born-Infeld type field, Sov. Phys. JETP 23 (1966) 861.

[10] R. Courant, Dirichlet's principle, conformal mapping and minimal surfaces, Interscience Pub. Inc., New York U.S.A. (1950).

[11] Y.L. Xin, Minimal submanifolds and related topics, Nankai Tracts in Mathematics 8, World Scientific, Singapore (2003). 
[12] U. Dierkes, S. Hildebrandt, R. Jakob, F. Sauvigny and A. Kèuster, Minimal surfaces, Springer-Verlag, Berlin-Heidelberg Germany (2010).

[13] R. Finn, On equations of minimal surface type, Ann. Math. 60 (1954) 397.

[14] M.H.L. Pryce, The two-dimensional electrostatic solutions of Born's new field equations, Proc. Cambr. Phil. Soc. 31 (1935) 50.

[15] M.H.L. Pryce, On a uniqueness theorem, Proc. Cambr. Phil. Soc. 31 (1935) 625.

[16] R. Ferraro, 2D Born-Infeld electrostatic fields, Phys. Lett. A 325 (2004) 134 [hep-th/0309185] [INSPIRE].

[17] R. Ferraro and M.E. Lipchak, Born-Infeld corrections to Coulombian interactions, Phys. Rev. E 77 (2008) 046601 [hep-th/0609141] [INSPIRE].

[18] R. Ferraro, Born-Infeld electrostatics in the complex plane, JHEP 12 (2010) 028 [arXiv: 1007.2651] [INSPIRE].

[19] O. Kobayashi, Maximal surfaces in the 3-dimensional Minkowski space $L^{3}$, Tokyo J. Math. 6 (1983) 297.

[20] G. Boillat, Nonlinear electrodynamics - Lagrangians and equations of motion, J. Math. Phys. 11 (1970) 941 [INSPIRE].

[21] J. Plebański, Lectures on non-linear electrodynamics, NORDITA, Copenhagen Denmark (1970).

[22] I. Bialynicki-Birula, Nonlinear electrodynamics: variations on a theme by Born and Infeld, in J. Lopuszanski's Festschrift, Quantum Theory of Particles and Fields, B. Jancewicz and J. Lukierski eds., World Scientific, Singapore (1983), pg. 31.

[23] R. Kerner, A.L. Barbosa and D.V. Gal'tsov, Topics in Born-Infeld electrodynamics, in Proceedings of XXXVII Karpacz Winter School, J. Lukierski and J. Rembielinski eds., Amer. Math. Soc., Providence U.S.A. (2001) [AIP Conf. Proc. 589 (2001) 377] [inSPIRE].

[24] M. Aiello, G. Bengochea and R. Ferraro, Anisotropic effects of background fields on Born-Infeld electromagnetic waves, Phys. Lett. A 361 (2007) 9 [hep-th/0607072] [INSPIRE].

[25] E.Yu. Petrov and A.V. Kudrin, Exact self-similar solutions in Born-Infeld theory, Phys. Rev. D 87 (2013) 087703 [arXiv:1302.4353] [INSPIRE].

[26] M.K.-H. Kiessling, Convergent perturbative power series solution of the stationary Maxwell-Born-Infeld field equations with regular sources, J. Math. Phys. 52 (2011) 022902 [INSPIRE].

[27] M.K.-H. Kiessling, Some uniqueness results for stationary solutions to the Maxwell-Born-Infeld field equations and their physical consequences, Phys. Lett. A 375 (2011) 3925.

[28] Y. Brenier, Hydrodynamic structure of the augmented Born-Infeld equations, Arch. Rat. Mech. Anal. 172 (2004) 65.

[29] T. Dereli and R.W. Tucker, Charged relativistic fluids and non-linear electrodynamics, Europhys. Lett. 89 (2010) 20009 [arXiv:1001.1282] [INSPIRE].

[30] D. Burton, R. Trines, T. Walton and H. Wen, Exploring Born-Infeld electrodynamics using plasmas, J. Phys. A 44 (2011) 095501 [arXiv:1006.2246] [inSPIRE].

[31] R. Ferraro, Testing Born-Infeld electrodynamics in waveguides, Phys. Rev. Lett. 99 (2007) 230401 [arXiv:0710.3552] [INSPIRE].

[32] R. Ferraro, Testing nonlinear electrodynamics in waveguides: the effect of magnetostatic fields on the transmitted power, J. Phys. A 43 (2010) 195202 [INSPIRE]. 